\title{
Anabases
}

ANABASES Traditions et réceptions de l'Antiquité

29 | 2019

Varia

\section{Les Horti Tauriani de Pierre Grimal ou les prémices des Jardins romains}

Eric Morvillez

\section{(2) OpenEdition}

Journals

Édition électronique

URL : https://journals.openedition.org/anabases/9301

DOI : 10.4000/anabases.9301

ISSN : 2256-9421

Éditeur

E.R.A.S.M.E.

Édition imprimée

Date de publication : 14 avril 2019

Pagination : 301-312

ISSN : 1774-4296

\section{Référence électronique}

Eric Morvillez, «Les Horti Tauriani de Pierre Grimal ou les prémices des Jardins romains », Anabases [En ligne], 29 | 2019, mis en ligne le 14 avril 2021, consulté le 06 novembre 2021. URL : http:// journals.openedition.org/anabases/9301 ; DOI : https://doi.org/10.4000/anabases.9301

(c) Anabases 

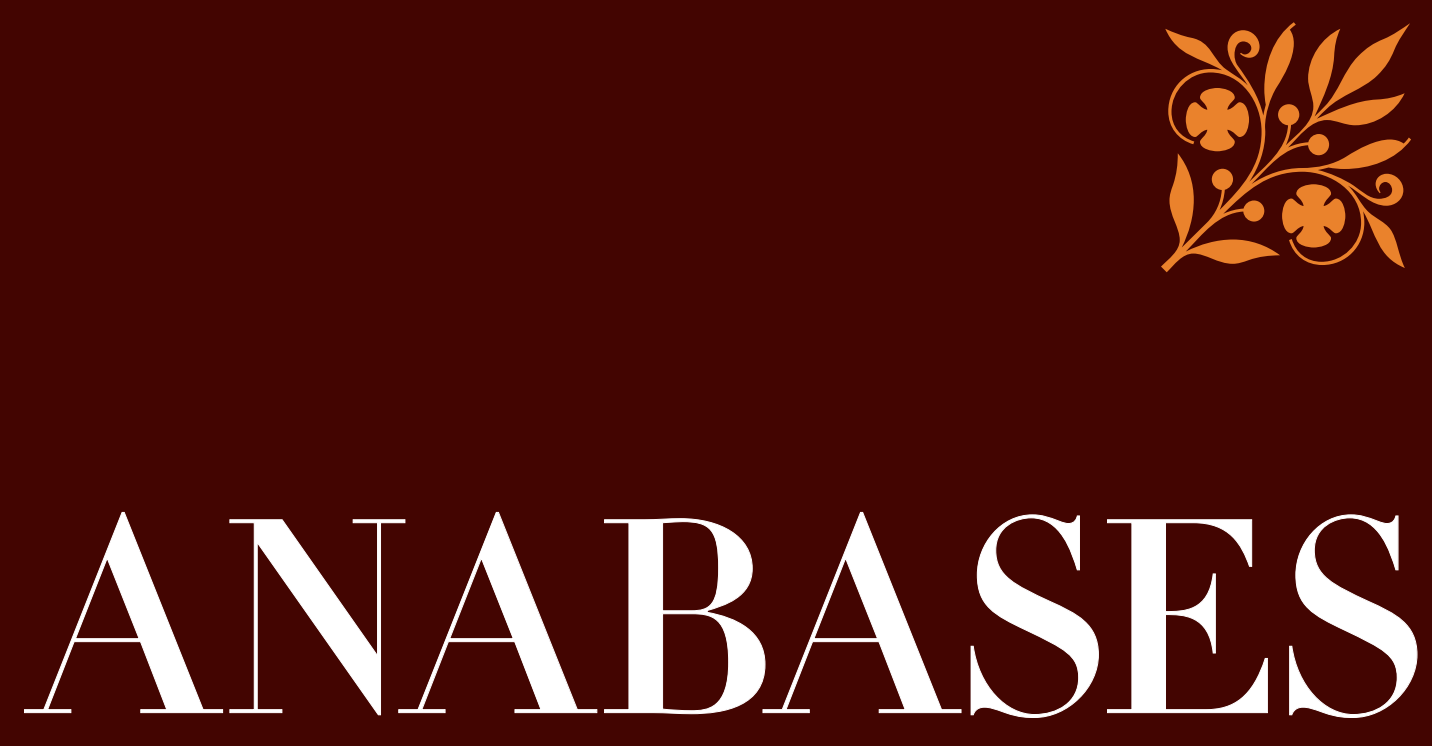

Traditions et Réceptions de l'Antiquité

\section{No29 \\ 2019}

Juliette Ernst Sculptures onctueuses de Meekyoung Shin Théâtre antique et travaux savants dans la Première modernité L'Antiquité dans la peinture (17911880) Réception d'Ovide Pierre Grimal 
ANABASES

Traditions et Réceptions de l'Antiquité

Revue de l'équipe de recherche E.R.A.S.M.E.

Université Toulouse-Jean Jaurès (UT2J)

Anabases dispose d'un Comité de lecture international. Chaque article envoyé à la rédaction est soumis, une fois anonymisé, à l'expertise de deux spécialistes qui rendent un rapport écrit. Les deux rapports anonymisés sont transmis à l'auteur qui tient compte des observations en vue de la publication.

\section{Comité SCIEnTIFIQUe}

Germaine Aujac (université Toulouse-Jean Jaurès : histoire de la géographie et des sciences antiques)

Florence Bouchet (université Toulouse-Jean Jaurès : littérature médiévale)

Hinnerk BruHns (CNRS : histoire économique et sociale ancienne et contemporaine)

Paulo Butti de Lima (université de Bari : historiographie et réception de l'Antiquité)

Luciano CANFora (université de Bari : littérature et histoire anciennes, historiographie)

Giovanna Ceserani (Stanford University : histoire intellectuelle et historiographie de la tradition classique)

Temístocles Cezar (université de Porto Alegre : historiographie moderne)

Serafina Сиомо (University of London, Birkbeck College : histoire des mathématiques et des sciences)

Paul Demont (université de Paris Sorbonne : philologie grecque et héritage classique)

Marie-Laurence Desclos (université de Grenoble II : philosophie de l'Antiquité)

Olivier Devillers (université de Bordeaux 3 - Michel-de-Montaigne : littérature et historiographie latines)

Andrea Giardina (Istituto italiano di scienze umane : histoire du monde romain et de ses réceptions)

Ève Gran-Aymerich (aibl : histoire de l'archéologie et des transferts culturels)

François HaRTog (EHEss : historiographie ancienne et moderne)

Geneviève Hoffmann (université de Picardie : histoire des mondes grecs)

Christian JACOB (CNRS/EHEss : histoire comparée et épistémologie des savoirs)

Suzanne Marchand (Louisiana State University : histoire du classicisme et de l'orientalisme)

Wilfried Nippel (Humboldt Universität Berlin : histoire et historiographie de l'Antiquité)

Sylvie Pittia (université de Paris I-Panthéon Sorbonne : histoire et historiographie du monde romain)

Stéphane Ratri (université de Franche-Comté - Besançon : philologie et héritage latin)

Comité de RÉdaction

Jacques Alexandropoulos, Marielle de Béchillon, Corinne Bonnet, Laurent Bricault, Clément Bur,

Philippe Foro, Adeline Grand-Clément, Anne-Hélène Klinger-Dollé, Véronique Krings,

Thibaud Lanfranchi, Claudine Leduc, Pascal Payen, Grégory Reimond, Catherine Valenti

Éditeur RESPonsable

Pascal PAYen

Université Toulouse-Jean Jaurès (UT2J)

SECRÉTARIAT DE RÉDACTION

Anthony Andurand / Clément Bertau-Courbières / Corinne Bonnet / Clément Bur /

Adeline Grand-Clément / Anne-Hélène Kuinger-Dollé / Véronique Krings /

Catherine Valenti (université Toulouse-Jean Jaurès) / Noémie VillacÈQue (université de Reims)

Sites Web

http://plh.univ-tlse2.fr

Revues.org : http://anabases.revues.org

Aвonnement et vente aU numéro

Éditions De Boccard - 4, rue de Lanneau - 75005 Paris

info@deboccard.com - www.deboccard.com

Tél. : 0033/(0)143260037 - Fax : 0033/(0)143548583 




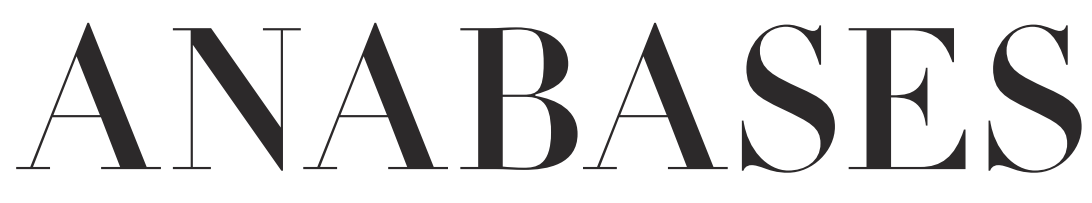

Traditions et Réceptions de l'Antiquité

$$
\begin{aligned}
& N \circ 29 \\
& 2019
\end{aligned}
$$

\section{E.R.A.S.M.E.}

Université Toulouse - Jean Jaurès 



\section{Sommaire}

$\mathrm{N}^{\circ} 29-2019$

\section{Historiographie et identités culturelles}

Ilse Hilbold

Les archives d'une bibliographe des sciences de l'Antiquité :

Juliette Ernst et la fabrique des relations internationales . . . . . . . . . I I3

Vivien LONGHI

La crise, une notion politique héritée des Grecs ? . . . . . . . . . . . 2I

Mireille Lacave-Allemand et Michel Lacave,

L’Antiquité dans la peinture en France, I79I-I880 :

une analyse quantitative à travers les Salons et les Prix de Rome . . . . . 37

Tiphaine Besnard

Du Weathering Project aux autoportraits en Venus :

Les sculptures onctueuses et savonneuses de Meekyoung Shin . . . . . 7 I

\section{Traditions du patrimoine antique}

Dossier dirigé par Pascale Paré-Rey et Malika Bastin-Hammou,

“La réception du théâtre antique dans les travaux savants de l’Europe

de la Première modernité »

Malika Bastin-Hammou et Pascale Paré-Rey

“ La réception du théâtre antique dans les travaux savants

de l'Europe de la Première modernité » . . . . . . . . . . . . . . . . 89

Kevin Bovier

Rétablir la métrique de Térence au $\mathrm{XvI}^{\mathrm{e}}$ siècle :

le cas du Iudicium de Glaréan (1540) . . . . . . . . . . . . . . . . . . . . . . 93 
Brice Denoyer

L'héritage de la métrique antique

dans l'alexandrin français au xvie siècle . . . . . . . . . . . . . IO7

Giovanna Di Martino

Vittorio Alfieri's tormented relationship with Aeschylus:

Agamennone between Tradition and Innovation . . . . . . . . . . . . . . . I2I

Marco Duranti

La condanna del prologo diegetico euripideo dagli scoli antichi

ai trattati del Cinquecento . . . . . . . . . . . . . . .

Rosario López Gregoris

L'influence de l' Arte nuevo de hacer comedias de Lope de Vega

dans l'usage des modèles classiques latins en Espagne

pendant le Siècle d'or et le Baroque » . . . . . . . . . . . . . . . . I49

Cressida Ryan

Sophoclean scholarship as a tool

to interpret eighteenth-century England . . . . . . . . . . . . . . г 6 I

Záviš ŠumaN

Axiologie critique de La Mesnardière . . . . . . . . . . . . . . . . . . . . I79

\section{Archéologie des savoirs}

Dossier dirigé par Cristina Noacco

“2000 ans déjà... Aspects de la réception d'Ovide » . . . . . . . . . . . I93

\section{La réception d'Ovide au Moyen Âge}

Jean-Marie Fritz et Cristina NoAcco

Lire Ovide au xiI ${ }^{\mathrm{e}}$ siècle : Arnoul d'Orléans

commentateur des Métamorphoses . . . . . . . . . . . . . . . $\quad{ }_{195}$

Franck Coulson

Le mythe de Pythagore dans le commentaire

“Vulgate » des Métamorphoses . . . . . . . . . . . . . . . . . . . . . . 2I

Marylène Possamaï

Comment éditer l'Ovide moralisé :

le problème de la mise en page du manuscrit Rouen Bm O.4 . . . . . . . 225

Anneliese Pollock Renck

Les Hérö̈des à la fin du Moyen Âge : pour une définition élargie de l'acte traducteur . . . . . . . . . . . . . . . . . . 239 
II. La réception d'Ovide à l'époque moderne

Fátima Díez Platas et Patricia Meilán Jácome

Le poète dans son œuvre. Ovide dans les images des Fasti

et des Tristia entre les Xv et $\mathrm{xvI}^{\mathrm{e}}$ siècles . . . . . . . . . . . . . . . . . 255

Ana Paula Rebelo Correia

Les représentations des Métamorphoses d'Ovide

dans les azulejos portugais. Influence des modèles gravés français . . . . 269

Sarah ReY

Figures d'Orphée au cinéma . . . . . . . . . . . . . . . . . . . 277

\section{Actualités et débats}

Marine LE BAIL

La modernité littéraire serait-elle affaire d'Antiquité(s) ?

Euvres \& Critiques: La contribution de l'archéologie à la genèse

de la littérature moderne, XLII, I, René Sternke dir., 20I7, 338 p. . . . . . . 2 29I

\section{Lire, relire la bibliothèque des sciences de l'Antiquité}

Éric Morvillez

“Les Horti Tauriani de Pierre Grimal

ou les prémices des Jardins romains » . . . . . . . . . . . . . . . . 30I

Pierre Grimal

“Les Horti Tauriani. Étude topographique sur la région

de la Porte Majeur ",MEFRA, tome 53, rg36. p. 25o-286 . . . . . . . . . . . 3 3i3

\section{L'atelier de l'histoire : chantiers historiographiques}

L'Antiquité au musée (coordonné par Adeline Grand-Clément) (6)

Aurélie Rodes, Catherine Valenti

Les Gaulois au musée . . . . . . . . . . . . . . . . . . . .

355

L'Atelier des doctorants (coordonné par Adeline Grand-Clément) (16)

Andrea Avalli

La question étrusque dans l'Italie fasciste $\ldots \ldots \ldots$. . . . . . . 360 
Droit et réception de l'Antiquité

(coordonné par Marielle de Béchillon et Hélène Ménard) (6)

Entre Clio et Thémis. Entretien avec Dario Mantovani, réalisé par

Hélène Ménard (Maître de Conférences d'Histoire romaine, à l'Université

Paul Valéry - Montpellier III), le 22 juin 20I8, à l'occasion de la parution

aux Belles Lettres du livre Les juristes écrivains de la Rome antique.

Les ouvres des juristes comme littérature (juin 20ı8) et de la création

de la chaire “ Droit, culture et société de la Rome antique »

au Collège de France $\left(\mathrm{I}^{\mathrm{er}}\right.$ novembre $\left.20 \mathrm{I} 8\right) \ldots$. . . . . . . . . . . . .

\section{Comptes rendus}

Philippe Borgeaud et Sara Petrella

Le singe de l'autre.

Du sauvage américain à l'histoire comparée des religions (A. Guedon) . . $\quad 37 \mathrm{I}$

Roberta Casagrande-Kim, Samuel Thrope et Raquel Ukeles (éd.)

Romance and reason. Islamic transformations of the classical past

(Cl. Bertau-Courbières) . . . . . . . . . . . . . . . . . . . . .

Hinnerk BruHns

Max Webers historische Sozialökonomie.

L'économie de Max Weber entre histoire et sociologie (Th. Lanfranchi) . . 374

Andrea Cozzo

Riso e sorriso, e altre saggi sulla nonviolenza nella Grecia antica,

(Fr. Pr. Barone) . . . . . . . . . . . . . . . . . . . 377

Franz Cumont

Manichéisme (St. Ratti) . . . . . . . . . . . . . . . 378

Emmanuelle HÉnin et Valérie NAAs (dir.)

Le mythe de l'art antique (Cl. Evrard) . . . . . . . . . . . . . . . . . 380

Jacques Jouanna, Henri Lavagne, Alain Pasquier,

Véronique SchiLtz et Michel Zink (éd.)

Au-delà du Savoir : Les Reinach et le Monde des Arts (G. Hoffmann) . . . .

382

Mario Liverani

Imagining Babylon: The Modern Story of an Ancient City (C.Bonnet) . . . 386

Françoise-Hélène Massa-Pairault, Claude Pouzadoux (Dir.)

Géants et Gigantomachie entre Orient et Occident (C.Giovénal) . . . . . . .

Scott McGill, Joseph Pucci (éd.)

Classics renewed. Reception and Innovation in the Latin Poetry

ofLate Antiquity (S. Clément-Tarantino) . . . . . . . . . . . . . . . . . . . 
Maxwell T. PAule

Canidia, Rome's First Witch (C. Landrea) . . . . . . . . . . . . . . . . . . . 39г

Jessica Priestley, Vasiliki Zali (éd.)

Brill's Companion to the Reception of Herodotus in Antiquity

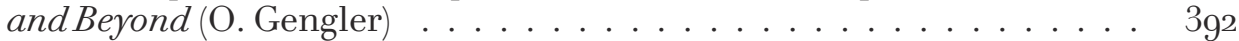

Salvatore QuAsimodo

La Lyre grecque $(\mathrm{M}$. Bianco) . . . . . . . . . . . . . . . . . . 395

Brett M. Rogers, Benjamin Eldon Stevens (éd.)

Classical Traditions in Modern Fantasy (M. Scapin) . . . . . . . . . . . . . 397

Maria Teresa Schettino et Céline UrLacher-Becht (dir.)

Ipse dixit. L'autorité intellectuelle des Anciens : affirmation,

appropriations, détournements (C. Psilakis) . . . . . . . . . . . . . 398

Guy G. Stroumsa

Religions d'Abraham : histoires croisées (D. Lorin) . . . . . . . . . . . . 400

Jean Yvonneau (éd.)

La Muse au long couteau. Critias, de la création littéraire

au terrorisme d'État (G. Hoffmann) . . . . . . . . . . . . . . 405

Résumés . . . . . . . . . . . . . . . . . . . . . 409

Index .............................. 423 



\section{Lire, relire la bibliothèque des sciences de l'Antiquité}



Anabases 29 (2019), p. 301-312.

\section{Les Horti Tauriani de Pierre Grimal ou les prémices des Jardins romains}

Eric MorviLlez

Commentaire de « Les Horti Tauriani, Étude topographique sur la région de la Porte Majeure » in MEFRA 53 (1936), p. 250-286.

n 1936, l'École française de Rome faisait paraître une longue synthèse
sous la plume du jeune Pierre Grimal, membre de l'institution depuis
un an. C'est à l'âge de 24 ans, avec une maturité indéniable, que le jeune homme propose cet article dédié à la colline des jardins, l'Esquilin, pour brosser plus précisément une synthèse critique des connaissances sur les Horti Tauriani. Rappelons tout d'abord que ce célèbre parc, propriété au $\mathrm{I}^{\mathrm{er}}$ siècle de T. Statilius Taurus, consul en 44, passa dans propriété impériale, après la condamnation pour magie de son propriétaire, sous Claude, à l'instigation d'Agrippine comme nous l'apprend Tacite'. Ces horti, qui s'étendaient sur une surface estimée actuellement à près de 36 hectares, ont une histoire complexe et discutée : s'attacher à ce dossier n'était pas sans difficultés. Prenant ce « morceau de banlieue qui hésite entre Rome et campagne, avec ses jardins entrecoupés de chemins creux et de murs ${ }^{2}$, Pierre Grimal s'attachait à restituer la topographie de cette vaste région de l'Vrbs. Mais s'il s'y était attelé, c'était pour trouver, comme il le dit, la solution à des questions topographiques non résolues, mais aussi pour d'autres raisons scientifiques comme nous allons le découvrir plus loin. Il propose ici ses premières conclusions sur une zone excentrée, moins commentée, où pas moins de onze noms de jardins

1 Tacite, Annales, XII, 59.

2 P. Grimal, “Les Horti Tauriani, Étude topographique sur la région de la Porte Majeure », MEFRA, 53, 1936, p. 252. 
différents sont connus. Les notices sur ces derniers restaient, comme P. Grimal le souligne au début de son texte, très factuelles, peu développées depuis les bilans menés lors des grandes fouilles entreprises avec le renouveau de Rome à la fin du $\mathrm{XIX}^{\mathrm{e}}$ siècle, à la faveur de l'unité politique de l'Italie. C'est Rodolfo Lanciani qui avait écrit une synthèse des découvertes effectuées entre 1873 et 1874 dans le quartier ${ }^{3}$. Rappelons que le domaine était délimité au temps d'Auguste par la via Labicana, l'ancien mur servien et le futur tracé du rempart d'Aurélien. Une zone fut ensuite rattachée aux célèbres Horti Lamiani ${ }^{4}$. Une grande partie des jardins de la colline fut réunie sous Gallien, pour être au $\mathrm{Iv}^{\mathrm{e}}$ siècle annexée aux Horti Liciniani voisins.

Rentré à l'École Normale Supérieure en 1932, Pierre Grimal avait été reçu 3e à l'agrégation de Lettres classiques en 1935, puis rentrait comme membre à l'École Française de Rome, pour y séjourner entre 1935 et 1937. C'est pendant son séjour romain, en contact direct avec la Ville éternelle et le terrain, qu'il mène son enquête. Il compose ce travail de topographie historique, confrontant méthodiquement les sources textuelles, épigraphiques et archéologiques aux courbes du terrain qu'il a arpenté et aux vestiges aperçus et discutés par ses illustres prédécesseurs, étrangers et français.

Il n'est pas besoin de rappeler l'influence qu'ont eue par la suite Les Jardins romains à la fin de la République et aux deux premiers siècles de l'Empire, qui constituent son œuvre la plus célèbre, du moins la plus emblématique, sans doute la plus lue. Ces Jardins romains restent, sur ce sujet, l'œuvre de synthèse en français récurrente dans les bibliographies, même aujourd'hui. On notera que son auteur a encore été gratifié d'une citation en exergue d'une des sections de la grande exposition consacrée à l'art des jardins, au Grand Palais en 20175. Pas moins de trois autres éditions des Jardins se succèderont après la première en 1943 : celle de 1969, puis celle de 1984, avec une nouvelle préface qui en dit long sur la maturation de l'œuvre, et enfin la dernière en 2008, après la disparition de leur auteur en 1996. Or, on sait que ce livre se décompose en fait en quatre traités qui

3 W. Henzen et R. Lanciani, “ Delle scoperte principali avvenute nella prima zona del nuovo quartiere Esquilino », $B C A R, 2,1874$, p. 42-61.

4 M. Cima, E. La Rocca, Le tranquille dimore degli dei : la residenza imperiale degli Horti Lamiani. Catalogo della mostra, Venise, 1986. Sur les Horti Tauriani, on consultera, en plus des articles mentionnés infra, la notice de E. PAPI, s.v. Horti Tauriani, in E. M. Steinby (a cura di), Lexicon Topographicum Urbis Romae, III, 1996, p. 85 ; D. Mancioli, “ Horti Tauriani », in G. Pisani Sartorio, L. Quilici (a cura di), L'archeologia in Roma Capitale tra sterro e scavo. Roma Capitale 1870-1911, Rome, 1983, p. 261 ss, M. Cima, E. Talamo, Gli horti di Roma antica, Milan, 2008, p. 93-97.

5 L. Le Bon, M. Jeanson et C. Zellal (dir.), Jardins. Exposition Grand Palais - Galeries nationales, 15 mars - 24juillet 2017, Paris, RMN, 2017. 
en forment les quatre parties ${ }^{6}$. L'article qui nous intéresse ici en constitua donc, $a$ priori sans le savoir, une première ébauche pour le chapitre de topographie dédié aux jardins de Rome. Les conclusions n'en sont d'ailleurs pas fondamentalement différentes ${ }^{7}$. Je souhaite montrer ici combien dans cette synthèse se trouvent déjà ancrés la méthode et les principes de l'auteur, sa manière d'entremêler les thématiques et les différentes disciplines, pour restituer une vue d'ensemble. On peut saisir la gestation extrêmement précoce d'une façon de faire qu'il a appliquée, progressivement, à l'ensemble de ses problématiques, pour réaliser l'essai final. Il me semble que, dans cet article de 1936, se trouvent les prémices du livre de 1943 : croisement systématique de tous les types de sources, goût pour la topographie, interrogations critiques avec réponses nuancées, remise en cause des thèses anciennes. L'ensemble reste nuancé d'une sage prudence, d'arguments pesés, avec un souci constant de rendre à chacun sa pensée. Mais il fait preuve d'une certaine audace pour un tout jeune membre de l'École Française de Rome, face aux théories des plus grands maîtres en matière de topographie romaine. On y sent enfin la filiation latine de son maître Jean Bayet, à l'origine du choix du sujet des jardins ${ }^{8}$ - même si la coloration religieuse est la moins évidente ici, compte tenu du sujet - , mais aussi l'admiration pour les théories de Jérôme Carcopino, citée dans l'article à propos de la basilique pythagoricienne de la Porte Majeure?

Mais observons d'abord le contexte de la rédaction de cet article. Il prend forme en même temps que le travail d'édition du De Aquaeductu de Frontin auquel s'est attelé P. Grimal et qui porte sur la gestion des aqueducs de la ville de Rome ${ }^{10}$. Il y manie à l'identique le croisement permanent des sources et de la topographie, mais aussi la confrontation de la matérialité du terrain aux textes latins, aux divers manuscrits discutés, traduits et commentés. La réalité des ruines s'y marie à l'érudition textuelle, avec un aller-retour permanent entre vestiges et bibliothèque. La parution aux Belles Lettres de sa traduction en 1944 sera marquée par une série de comptes rendus qui souligneront les qualités qui se font jour dans l'article de 1936. On salue en particulier la carte des aqueducs de Rome, placée en fin de volume. C'est cette transdisciplinarité que souligne

$6 \quad 2^{\text {e }}$ partie, p. 107-210, ce que souligne déjà Henri-Irénée Marrou, “ Compte rendu de Pierre Grimal, Les jardins romains ", REA, 46, 1944, p. 192 ; S. Rey, Écrire l'histoire ancienne à l'École Française de Rome (1873-1940), Rome, EFR, BEFAR 462, 2012, p. 117.

7 P. Grimal, Les jardins romains, Paris, Fayard,1984 (3e édition), p.145-154, en particulier les p. 150-151.

8 N'oublions pas ici le sous-titre, fondamental : “ Essai sur le naturalisme romain ».

9 Grimal, Horti Tauriani, p. 250, n. 5 et p. 251.

10 Cf I. A. Richmond, “ Compte rendu de Frontin, Les aqueducs de la ville de Rome. Texte établi, traduit et commenté par P. Grimal (1944) », JRS, 36, 1946, p. 219. 
déjà Pierre Boyancé, dans son compte rendu de la traduction de Pierre Grimal

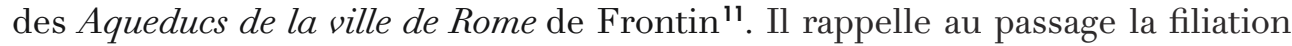
de l'élève avec son maître, mais aussi les mérites de la méthode, appliquée à la traduction du traité de Frontin comme à la synthèse des Horti Tauriani : “ l'étude d'un tel traité touche à la fois à la philologie, à l'histoire, à l'archéologie et au droit. Et même si ce n'est pas la moindre difficulté du travail, il fallait s'initier quelque peu à l'hydraulique. L'auteur, élève de M. Jean Bayet, s'est montré égal à toutes ces tâches. La traduction manuscrite, très imparfaite, oblige l'éditeur à intervenir avec décision ; M. Grimal n'y a pas manqué ${ }^{12}$... L'auteur des Jardins romains a résolu plus d'un problème de topographie ». Pierre Grimal lui-même considère que « son aide principale sera le petit livre de Frontin ${ }^{\prime 3}$. Dans les Horti Tauriani, pas moins de 4 pages (p.258-61) sont consacrées à affiner le tracé des conduites d'eau dont dépend celui des jardins. Ce sont donc bien les aqueducs et la réflexion sur leur usage et leur concentration au nord-est de Rome qui ont amené Pierre Grimal à s'intéresser aux jardins romains.

On remarque en revanche que les figures sont rares : 3 plans et 2 photographies, d'assez mauvaise qualité, mais aucun plan ancien de Rome, aucune image de statue - pourtant essentielle pour ce dossier - ne sont reproduites. Cette pauvreté de l'illustration restera d'ailleurs par la suite l'un des regrets récurrents de l'auteur. Dans la préface de la $3^{\text {e }}$ édition des Jardins romains, soulignant les avancées remarquables en matière pompéienne de sa collègue Wilhelmina Jashemski, il se plaindra du manque d'images de la nouvelle version des Jardins et de la complexité encore d'obtenir des clichés, mais aussi d'acquérir leurs droits ${ }^{14}$.

Mais attachons-nous maintenant à quelques points qui touchent autant aux problématiques abordées qu'aux méthodes mises en œuvre. Si l'étude des aqueducs de Frontin a poussé Grimal à s'intéresser aux horti, il faut souligner sa prédisposition à choisir des dossiers topographiques. Avec comme but poursuivi

1 P. BoyancÉ, “Compte rendu de Frontin, Les aqueducs de la ville de Rome, trad. P. Grimal », REA, 46, 1944, p. 363.

12 On peut le remarquer également dans l'article sur les Horti Tauriani, à propos de l'interprétation d'un passage de Strabon sur les noms des voies romaines du quartier : Grimal n'hésite pas à proposer sa lecture personnelle du texte (p. 256).

13 Grimal, Horti Tauriani, p. 250, dont il cite la traduction employée, celle de la collection Teubner de 1922.

14 Préface de la 3e édition des Jardins romains, 1984, p. IV. Elle restera une constante dans toutes les éditions de ce volume de Grimal, ce que soulignera sans ménagement Robert Étienne dans son compte rendu de la $3^{\mathrm{e}}$ édition : “ Oui, un livre toujours actuel, qu'on aurait aimé illustré de plans et de planches couleurs. Quant donc disposera-ton d'éditeurs assez audacieux et respectueux des savants qu'ils devraient honorer? » (REA, 89, 1987, p. 155). 
celui de donner une portée historique à sa démonstration. Ce qui l'intéresse dans les Horti Tauriani, c'est d'abord le sens des différents changements de propriétaires. En témoigne aussi son étude suivante, sur le Champ de Mars, publiée depuis Rennes à partir de 1941. Il y reconstitue une inscription très fragmentaire, dessine un plan (du même type que celui proposé pour les Horti Tauriani) et termine son étude par des considérations sur la politique urbaine d'Auguste, expliquant « pourquoi la plus grande partie de la presqu'île formée par le Tibre resta libre de constructions jusqu'à l'époque tardive : considérée comme dépendance des jardins d'Agrippa, elle était propriété légale du peuple romain, sur des titres récents et de bonne forme ${ }^{15}$. Ce lien entre topographie et commentaire épigraphique restera essentiel pour lui. Dans la fin de la préface de la 3 e édition des Jardins romains, il complètera encore par de nouvelles informations cet article sur la topographie du Champ de Mars et les constructions d'Agrippa, comme il le fait pour les Jardins de Pompée. Pour lui, les espaces plantés ont influencé l'univers politique romain autant que certains types de décors ou certaines œuvres phares, comme L'art d'aimer d'Ovide. Ses déductions matérielles sur les jardins débouchent sur une réflexion d'ordre historique: ainsi, le Palatin étant structurellement limité en surface, il voit dans la région $\mathrm{V}$ de l'Esquilin « un exemple de 'quartier royal', à la mode d'Alexandrie (...) Les parcs de la région $\mathrm{V}$ sont le dernier essai d'une résidence vraiment royale ${ }^{16}$ ». C'est à la fin de l'Antiquité, selon lui, avec Gallien puis Constantin que le projet trouve son aboutissement.

L'auteur est avant tout conscient de la place essentielle des aqueducs pour arroser les jardins et alimenter leurs nombreux édifices destinés à l'otium, ornés à l'envie de fontaines et nymphées. Gros consommateurs d'eau, les structures thermales étaient indispensables à ces espaces de plaisance où l'empereur, ses familiers et parfois la cour résidaient. Ce contrôle d'une eau saine et potable reste aussi un élément essentiel de la politique urbaine des empereurs, au même titre que le contrôle de l'annone. P. Grimal a vu dans la mainmise sur cette couronne de jardins - et plus particulièrement dans la région esquiline - une volonté impériale d'annexer en un seul domaine une zone associant contrôle utilitaire et espace de prestige, avec des édifices consacrés au loisir, partagé parfois avec la cour. C'est la “ thèse d'un jardin impérial unique » qu'il souhaite défendre ${ }^{17}$. Comme le soulignera ensuite Vincent Jolivet ${ }^{18}$, il existe bien un lien essentiel

15 P. Grimal, “ Agrippa et le Champ de Mars », RA, 19, 1942-43, p. 30.

16 Grimal, Horti Tauriani, p. 286.

17 Ibid., p. 280.

18 V. Jolivet, "Croissance urbaine et espaces verts à Rome ", in La Rome impériale, démographie et logistique. Actes de la table ronde de Rome, 25 mars 1994, Rome, EFR, BEFAR 230, 1997, p. 204. 


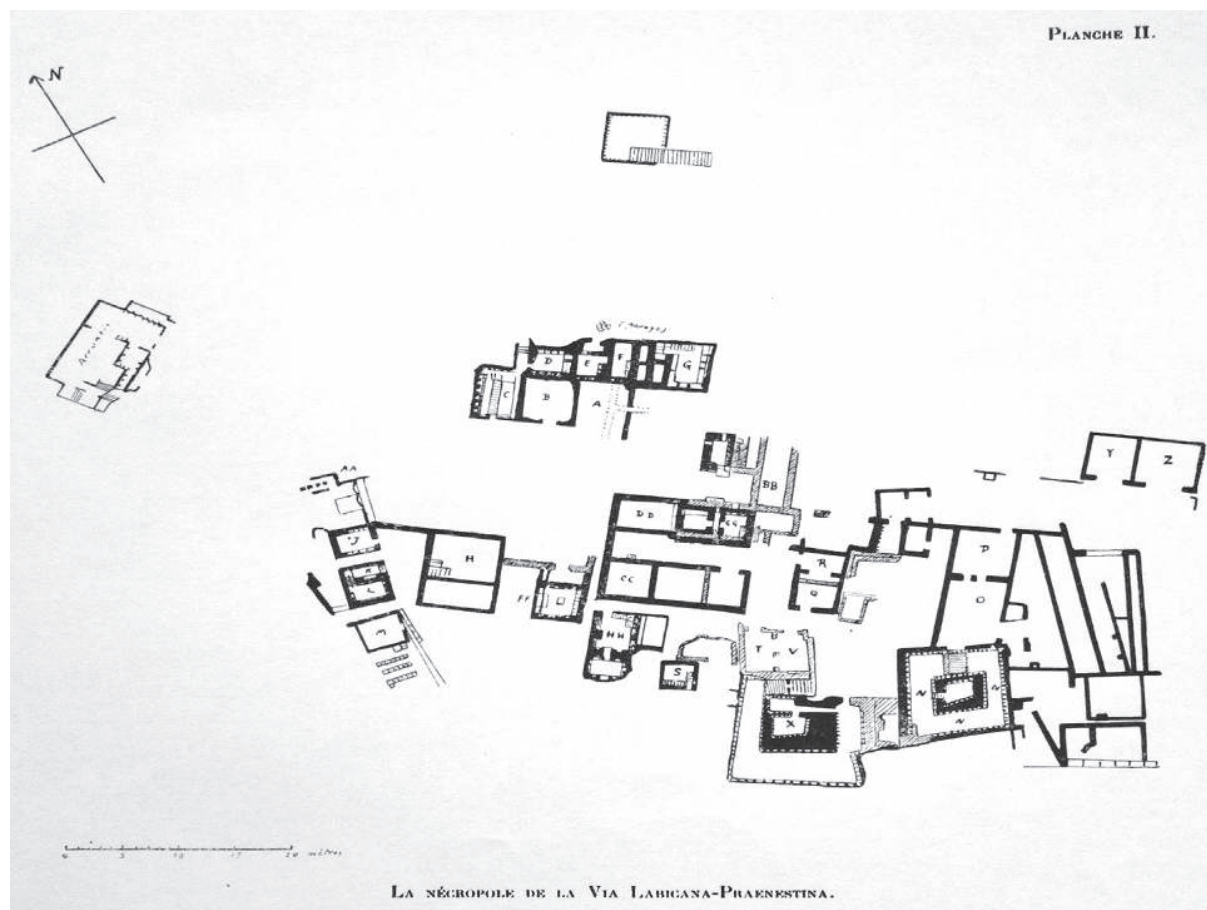

Fig.1. La nécropole de la Via Labicana-Praenestina, dans Grimal, Horti Tauriani, pl. II.

entre fiscus, contrôle des aqueducs et horti : « Si la politique impériale en matière de jardins trouvait ainsi sa justification dans l'intérêt public, l'empereur retirait également un ensemble d'avantages immédiat et considérable, de la constitution de la ceinture verte. Le secteur de l'Esquilin, l'un des premiers occupés par le Fisc, garantissait un contrôle direct sur les terminaux des aqueducs ».

Pour présenter son étude topographique, Grimal suit un plan partant «des lignes de forces du quartier: les aqueducs et les routes» (p. 253-258) pour traiter ensuite des questions de “localisations particulières» des différents parcs mentionnés dans les sources et inscriptions (le cœur de l'article, p. 258- 277, avec une étude détaillée de la zone de nécropole de la Via Labicana-Praenestina) (fig. 1). Puis il revient sur la moisson d'œuvres d'art découverte dans la zone (p. 277-280), pour achever sa démonstration avec la question des Horti Liciniani et la fonction de l'édifice à coupole dit de Minerva Medica (p. 280-285) ${ }^{19}$. Il s'arrête alors sur la

19 Sur cette question, voir M. Cima, “ Horti Liciniani », in S. Ensoli, E. La Rocca (a cura di) Aurea Roma. Dalla città pagana alla città cristiana, Rome, 2001, p. 97-103 et l'article de synthèse de F. Guidobaldi, “Horti nominis suis: considerazioni sulla residenza esquilina du Licinio Gallieno e sulla topografia degli Horti nell'area sud-est di Roma », 


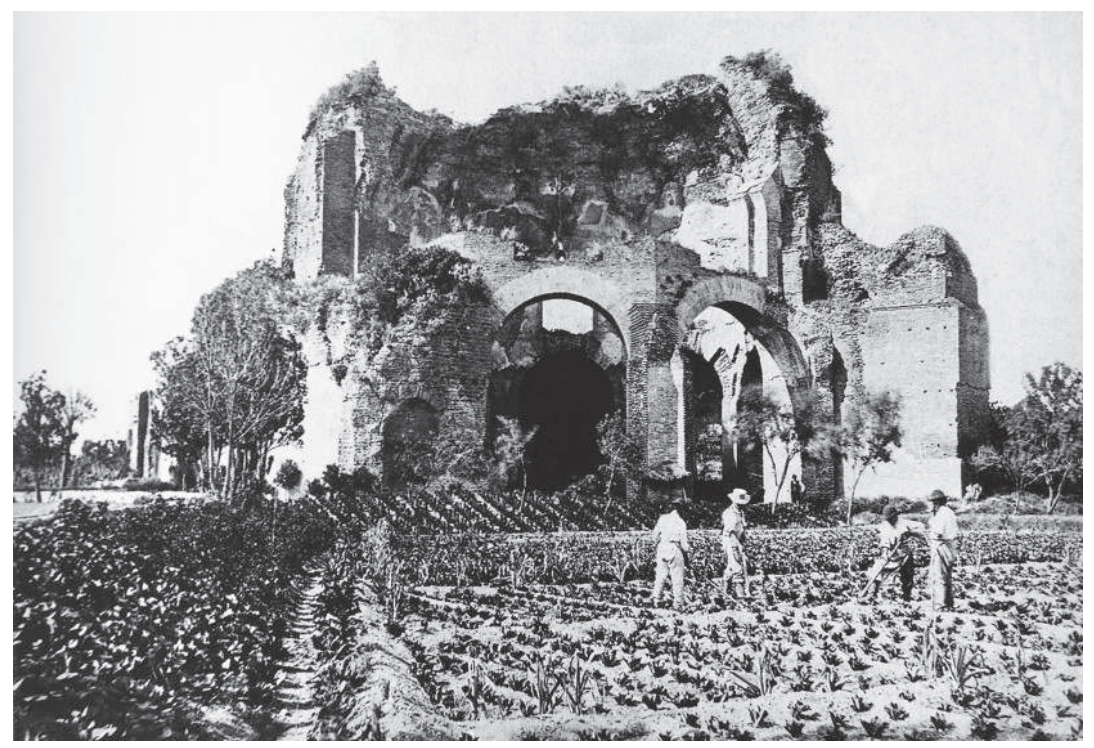

Fig. 2. Rome, le « temple de Minerva Medica » dans la zone

des Horti Liciniani, avant les travaux d'urbanisme de la fin du $\mathrm{XIX}^{\mathrm{e}}$ siècle dans Ensoli, La Rocca, Aurea Roma, p. 97, fig. 1.

fonction de cette salle à plan centré exceptionnelle, posée jusqu'au XIx ${ }^{\mathrm{e}}$ siècle dans la campagne (fig. 2) et qui fut rapidement emprisonnée entre les voies de chemin de fer qui aujourd'hui la défigurent. C'est la fonction d'un pavillon de plaisance qu'il privilégie, une sorte de diaeta - qu'il compare à la Piazza d'Oro de la Villa Hadriana pour la forme et à la villa de Toscane de Pline le Jeune pour ses installations de jeux d'eau. Il attire d'ailleurs l'attention sur la présence d'une conduite de plomb aménagée dans une des absidioles latérales qu'il a apparemment observée sur place (p. 284). Le monument sera présenté sous forme de maquette dans la grande exposition de la romanité de 1937 (fig. 3) 20. Il conclut dans les deux dernières pages sur le sens de cette concentration de parcs dans les mains impériales.

L'article est illustré d'un plan bicolore, soigneusement dessiné et annoté : le quartier moderne de l'Esquilin en rouge et les limites proposées et vestiges connus en noir (fig. 4). Cela annonce la méthode qui prévaudra dans les Jardins romains: photographies de vues aériennes contemporaines de la zone de

in Scritti in onore di Lucos Cozza, R. Coates-Stephens, L. Cozza (a cura di), LTUR, suppl. VII, p. 127-140, Rome, 2014, où l'article de Grimal est cité encore en bonne place.

20 Salle 42 de l'exposition (thermes, nymphées, aqueducs et fontaines), dans Mostra Augustea della Romanità, bimillenario della nascita di Augusto, 23 settembre 1937 23 settembre 1938, catalogo, quarta ed., 1938, p. 565-566, pl. CV. 


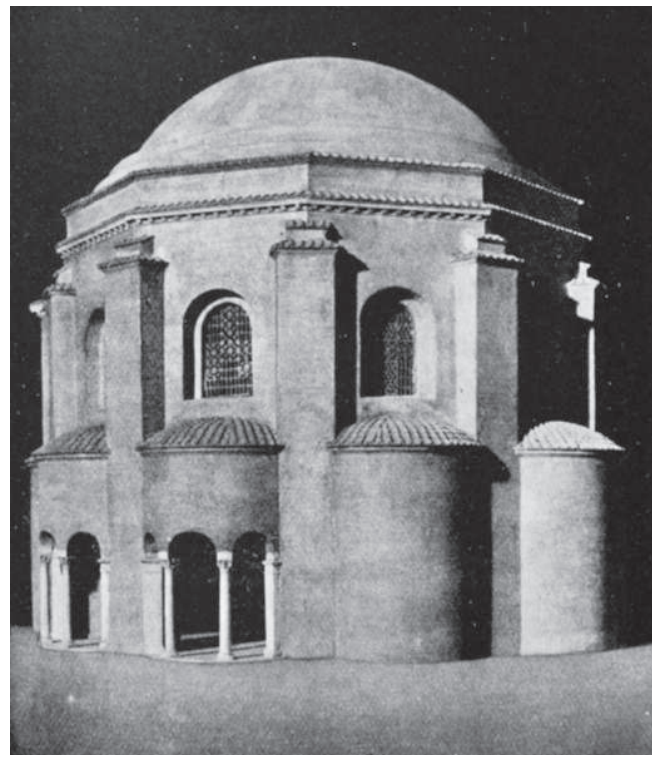

Fig 3. Maquette de l'édifice

dit de Minerva Medica,

d'après Mostra Augustea della Romanità, bimillenario della nascita di Augusto, 23 settembre 1937 - 23 settembre 1938, catalogo, quarta ed., Rome, 1938, pl. CV.

l'Esquilin, doublées d'un calque qui porte le tracé des restes antiques. Grimal reprend en cela en le " modernisant », le principe de superposition des plans antique et moderne de l'œuvre de Rodolfo Lanciani, mais avec une technique plus moderne, puisque le contour des jardins et la somme des connaissances de la synthèse apparaissent concentrées sur une feuille autonome. Un plan de Rome sur calque sera annexé à la future édition des Jardins romains, plan dont l'utilité fut saluée par les commentateurs ${ }^{21}$. Le procédé annonçait en quelque sorte les filtres et feuilles superposables de nos plans et cartes informatiques contemporains.

Mais quelle est la portée, ou du moins l'empreinte de cette étude de topographie romaine? Cette première synthèse situe l'auteur directement dans la lignée des grands travaux de cartographie antérieurs : ceux de G. Lugli, de R. Lanciani notamment, mais veut éviter l'écueil des dictionnaires épigraphiques ou monumentaux, où les entrées des jardins par propriétaires (comme dans le Platner-Ashby) restent très sommaires, énumérant sans vue d'ensemble, pavillons et vestiges, inscriptions et statues parfois séparées de leur contexte. Il cherche à prendre à bras le corps la complexité de l'histoire de ces jardins, puisqu'il souhaite démontrer l'idée, émise par J. Carcopino, que certains Horti se succèdent, d'autres s'absorbent, d'autres ne sont pas encore situés ${ }^{22}$. Il y avait un

21 “Retenons les faits d'ensemble, - dit H. I. Marrou - (qu'un transparent qui double le grand plan de Rome annexé au volume met commodément en évidence) »: cf. H. I. Marrou, “ Compte rendu de P. Grimal, Jardins romains », REA, 46, 1944, p. 194. Grimal, Horti Tauriani, p. 253, n. 4 en particulier. 


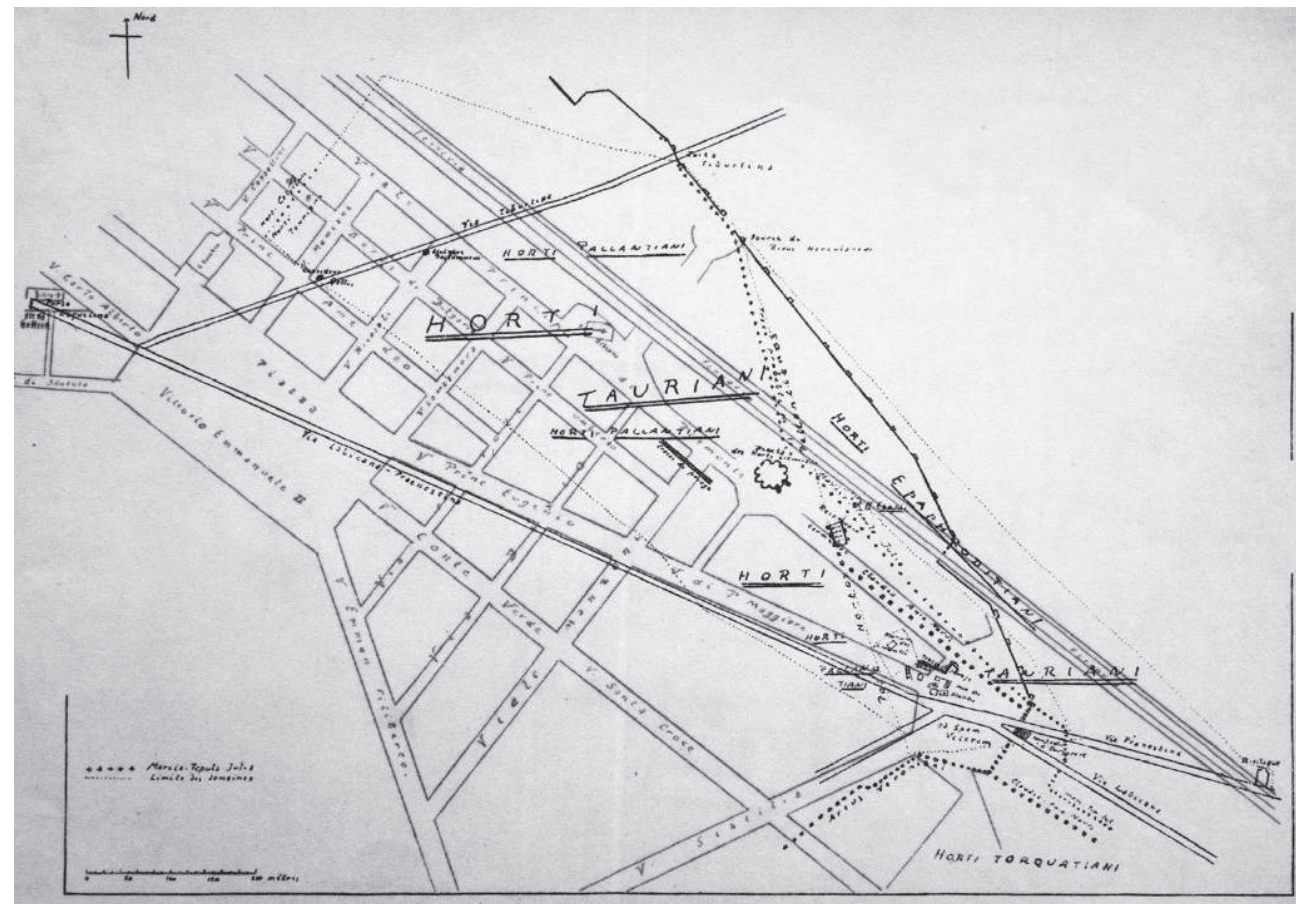

Fig. 4. Le quartier de la Porte majeure, d'après Grimal, Horti Tauriani, pl. I.

réel mérite, presque un défi, teinté d'une forme d'inconscience, à se lancer sur un tel sujet et à se mesurer directement aux grands maîtres du terrain de Rome que furent et Lugli et Lanciani, sans compter la somme du Platner-Ashby parue en 1929. Ce désir de faire le point est corroboré par un autre élément de méthode : P. Grimal, dès ce premier article, va rechercher des réponses, jusque dans les archives inédites de ses célèbres archéologues devanciers, faisant en même temps œuvre d'historiographe du sujet. On peut le voir par exemple à propos de l'Aqua Marcia, sur des fouilles anciennes, où Grimal confronte les théories de Parker publiées avec des documents de cahiers de fouilles extraits des archives Lanciani, à propos de l'interprétation de vestiges ${ }^{23}$.

Le défi fut payant et les méthodes systématiquement appliquées pour les Jardins romains. On peut citer plus tard, dans le long compte rendu sur ce dernier livre par Henri-Irénée Marrou, cette note révélatrice à propos de la deuxième partie du livre « dont les identifications topographiques demanderaient une discussion minutieuse: il y a là matière à retoucher une bonne trentaine d'articles du 
Platner-Ashby ${ }^{24}$ ». En ce sens, Grimal se positionnait déjà comme un successeur, mais aussi un contradicteur potentiel, comme il le sera avec Lugli sur la théorie de l'origine du jardin romain ${ }^{25}$. Plusieurs spécialistes français de la topographie romaine et de ses jardins, comme Jean-Pierre Guilhembet avec ses travaux sur les domus républicaines ${ }^{26}$, Vincent Jolivet pour les horti Luculliani ${ }^{27}$, ou encore Clément Chillet pour les jardins de Mécène ${ }^{28}$, ont planté de nouveaux jalons en partant de ce sillon ouvert. De nombreux spécialistes étrangers comme Kim Harswick pour les jardins de Salluste ${ }^{29}$ ou Nicholas Purcell pour la ceinture verte en général ${ }^{30}$, ont suivi cette méthode, sans oublier les représentants de l'école de Wilhelmina Jashemski, perpétuée par Katherine Gleason, Maureen Karoll, ou Amina Aïcha Malek, pour ne citer que quelques noms parmi tant d'autres dans la spécialité qu'est devenue l'archéologie des jardins. L’esprit impulsé par Pierre Grimal dans les Horti Tauriani puis les Jardins romains est celui aussi qui a inspiré des vocations, comme celles de Jean-Marie Pailler (autour des oscilla) ou Henri Lavagne, pour le thème des grottes, nourri des travaux comme ceux de Gilles Sauron, Hélène Dessales ou Pierre Vesperini, mais aussi des historiens des jardins d'autres périodes comme Monique Mosser ou Hervé Brunon, pour ne citer que quelques noms.

En conclusion, nous ne savons pas vraiment comment ce premier essai d'un jeune auteur encore débutant fut reçu, notamment par les archéologues italiens. Tout au plus doit-on souligner que l'article des Horti Tauriani est resté une base bibliographique toujours citée, à propos de ces fameux jardins, dans les synthèses, jusqu'à nos jours. Cet article, prometteur, était finalement une synthèse brillante, destinée à devenir la base d'un futur chapitre d'une œuvre en gestation, Les Jardins romains. Les conclusions de l'auteur sur le sujet varieront peu ensuite et sont encore aujourd'hui citées et discutées. Il reste qu'à l'époque, sur le plan méthodologique, pour le latiniste qu'était P. Grimal, le texte comme l'inscription priment encore : ils restent les documents fondamentaux et semblent conserver

24 Marrou, p. 194.

25 Sur cette question, cf. Rey, Écrire l'histoire p. 218.

26 J.-P. Guilhembet, “ La densité des domus et des insulae dans les XIV Régions selon les Régionnaires : représentations cartographiques ", MEFRA, 108, 1996, p. 7-26.

27 V. Jolivet, C. Sotinel, "Die Domus Pinciana. Eine kaiserliche Residenz in Rom », dans T. Fuhrer (dir.), Rom und Mailand in der Spätantike. Repräsentationen städtischer Räume in Literatur, Architektur und Kunst, Berlin-Boston, 2012, p. 137-160.

28 C. Chillet, De l'Étrurie à Rome. Mécène et la fondation de l'Empire, BEFAR 373, Rome, 2016.

29 K. Hartswick, The Garden of Sallust. A Changing Landscape, Austin, 2004.

30 N. Purcell, "The Horti of Rome and the Landscape of Property ", in Res bene gestae. Ricerche di storia urbana su Roma antica in onore di E. Margaret Steinby, Rome, 2007, p. 289-305. 
le pas sur des données archéologiques. Plusieurs fois dans le texte est clairement énoncée l'idée que tout est fait : qu'il n'y aura pas de reprise de fouilles, que l'on peut clore le dossier et qu'il est temps de faire le bilan ${ }^{31}$. Il y a là une forme de certitude de la part du jeune historien, qui voit dans les dossiers archéologiques un matériau "fermé ». Loin de moi l'idée de croire que P. Grimal n'était pas sensible aux realia. Bien au contraire. D'autres que moi ont souligné combien il revendiquait un " atavisme paysan »-qui a transparu jusque dans ses écrits plus personnels. Mais il appartient encore à une génération qui a l'habitude de voir l'archéologie comme une science auxiliaire de l'Histoire. Dans la préface de la

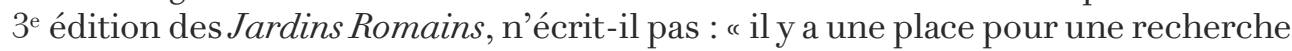
allant dans le sens de celle que nous avons poursuivie dans nos Jardins romains où le document factuel vient à l'appui du raisonnement, le confirme, mais ne le commande pas : un fait ne saurait jamais être que ponctuel, et une série de faits qu'un pointillé. Seules des considérations d'un autre ordre peuvent 'intégrer' cette masse informe ». Cette certitude ne sera ébranlée chez Pierre Grimal que progressivement. On en voit se manifester plus tard le doute et l'on sent combien il aurait aimé être le lecteur et l'utilisateur des volumes du corpus matériel des Gardens of Pompei que Wilhelmina Jashemski lui avait annoncés, parus en $1993^{32}$.

La tenue scientifique à travers le temps des Horti Tauriani en fait un article toujours utile ${ }^{33}$. Il constitue une étape indispensable pour comprendre l'historiographie des jardins romains. De cette étude émerge la personnalité de Pierre Grimal. Comme l'a écrit Marcel Renard, ce dernier a réussi à démontrer comment la création des jardins à Rome a pu représenter une «transposition artistique et concrète d'une culture ${ }^{34}$ ». Cet article précoce sur les Horti Tauriani contient en germe toutes les inflorescences des Jardins romains de Pierre Grimal, sa méthode autant que sa sensibilité.

Aujourd'hui, ce type de texte trouve son parallèle dans les cartes interactives sur le web qui permettent de placer informations et découvertes archéologiques.

31 Grimal, Horti Tauriani, p. 251 : « Il ne semble pas que d'ici longtemps le terrain puisse livrer de nouveaux documents dans ce quartier, l'un des plus populeux de Rome : l'ère des grandes trouvailles qui marquèrent la fin du siècle dernier (...) est maintenant bien close. Il est possible de dresser des index et de conclure. ”

32 W. F. Jashemski, The Gardens of Pompeii, Herculanum and the Villas destroyed by Vesuvius, volume II, New Rochelle - New York, 1993.

33 B. Germini, Statuen des strengen Still in Rom. Verwendung und Wertung eines grieschischen Stils im römischen Kontext, Rome, L'Erma di Bretschneider, 2008 s. v. Horti Tauriani, p. 80-82. Sur les collections de sculptures remployées dans des murs tardifs : R. Contes-Stephens, "Muri dei bassi secoli in Rome: observations on the reuse of statuary in walls found on the Esquiline and Caelian after 1870 », JRA, 14, 2001, p. 217-238.

34 M. Renard, “ Compte rendu de Pierre Grimal, Les Jardins romains », AC, 14, 1944, p. 227. 


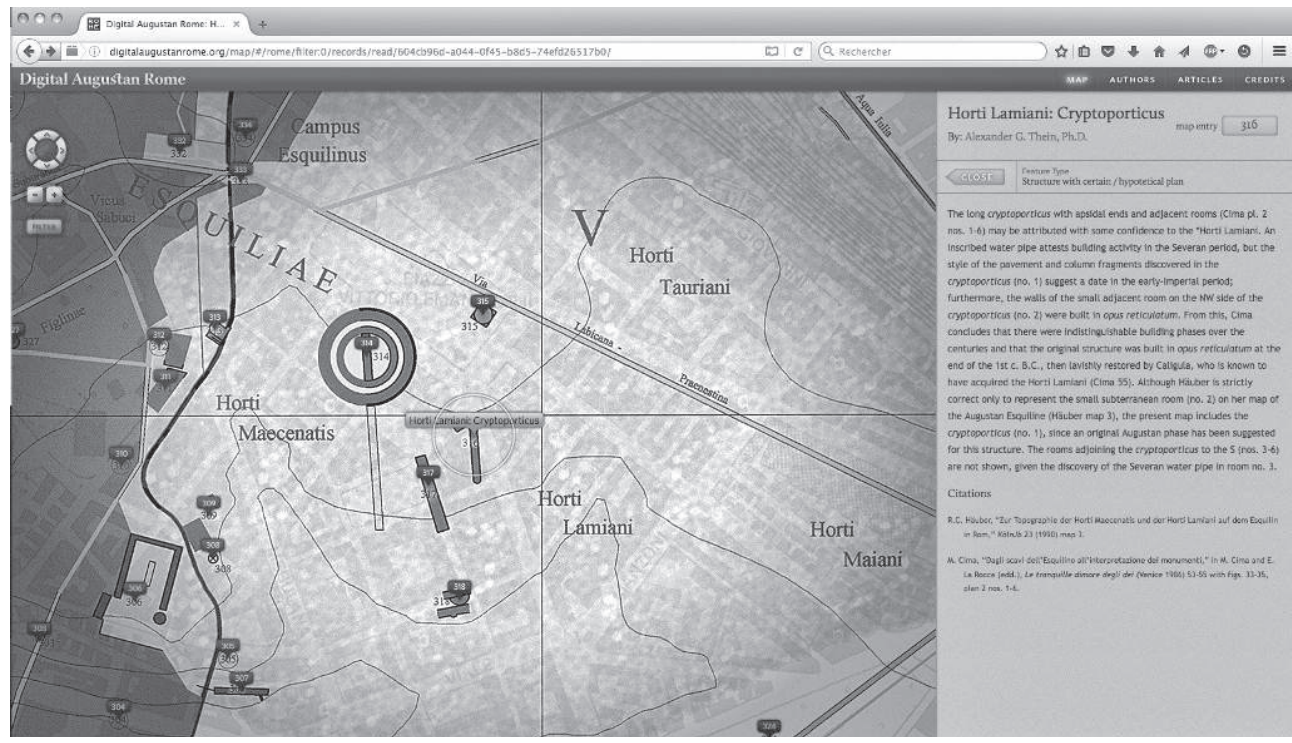

Fig. 5. Page de la carte de Rome du site Digital Augustan Rome (université d'Arizona) zone des jardins de l'Esquilin.

C'est le cas de la Carte de la Rome augustéenne publiée par l'université d'Arizona (Digital Augustan Rome) qui présente la Ville théoriquement en 14 ap. J.-C. et où figurent les Horti Tauriani (fig. 5). Si elle offre en quelques clics des notices synthétiques associées aux divers lieux et édifices recensés, elle est encore loin de permettre de rendre compte de la complexité des théories à l'œuvre dans un article de cette densité. On reste davantage au niveau des dictionnaires encyclopédiques. Ce type de média, s'il est évolutif et maniable, peut figer un état de la question à une époque donnée, risquant de tromper l'utilisateur sur la fragilité des hypothèses en présence, toujours susceptibles d'être remises en cause par une nouvelle documentation. Il serait intéressant de pouvoir intégrer à ces “ cartesbases de données » les études, anciennes et récentes des différents historiens et archéologiques, en hypertexte, pour fournir un instrument de travail critique à l'utilisateur. On mesure toute la difficulté pour matérialiser, comme l'avait tenté Pierre Grimal en 1936 dans sa propre carte, les limites des différents horti. Il reste qu'en comparant ces outils, on mesure, comme pour les modélisations 3D des villes antiques, les progrès possibles dans le champ des études de topographie historique.

\section{Eric MORVILLEZ}

Université d'Avignon et des Pays du Vaucluse eric.morvillez@univ-avignon.fr 